\title{
Comparative Analysis of Financial Network Topology for the Russian, Chinese and US Stock Markets
}

\author{
VLADIMIR BALASH \\ Saratov State University \\ Faculty of Mechanics and Mathematics \\ 83 Astrakhanskaya Street \\ RUSSIAN FEDERATION \\ ALEXEY FAIZLIEVSaratov State \\ UniversityRisk Institute \\ 83 Astrakhanskaya Street, RUSSIAN \\ FEDERATION \\ ALEXEY GRIGORIEV \\ Saratov State University \\ Faculty of Mechanics and Mathematics \\ 83 Astrakhanskaya Street \\ RUSSIAN FEDERATION
}

\author{
SERGEI SIDOROVSaratov State \\ UniversityRisk Institute \\ 83 Astrakhanskaya Street, RUSSIAN \\ FEDERATION
}

ALFIA CHEKMAREVA

Saratov State UniversityRisk Institute

83 Astrakhanskaya Street, RUSSIAN

FEDERATION

DMITRIY MELNICHUK

Saratov State University

Faculty of Mechanics and Mathematics

83 Astrakhanskaya Street

RUSSIAN FEDERATION

\begin{abstract}
This paper studies the properties of the Russian stock market by employing the data-driven science and network approaches. The theory of complex networks allows us to build and examine topological network structures of the market with the further identification of relationships between stocks and the analysis of hidden information and market dynamics. In this paper we will present an analysis of structural and topological properties of the Russian stock market using market graph, hierarchical tree, minimum spanning tree approaches. We compare topological properties of the networks constructed for the US and China stock markets with the properties of correspondingnetworksconstructedfortheRussianstockmarketusingadatasetspanningovereightyears.
\end{abstract}

Key-Words: Network analysis, Market graph, Degree distribution, Hierarchical tree, Minimum spanning tree

Received: November 7, 2019. Revised: March 9, 2020. Accepted: March 25, 2020. Published: March 31, 2020.

\section{Introduction}

To represent the interaction framework between agents in economic or financial systems, one can use network models. The definition of a financial network was given in [1], in which the nodes are various organizations, including banks, firms and investors, which are linked through a network of financial interdependencies, for example, cross-ownership of financial assets, inter-organizational debts / liabilities, social relations between board members and so on. A classic example of a banking (financial) network is built in [2], where it is illustrated how systemic risk can arise as a result of financial interdependencies be- tween banks. Most of real-world networks are constantly changing over time. The links between economic agents are usually activated or deactivated in dependence of the irregular interactions between the elements of the economic system. These activation templates may be sporadic and represent a huge role in the dynamics of network processes. One of examples of this situation is information spreading in social networks. The topology of network (heterogeneity in the connections, abundance of loops) plays an important role in the economic system dynamics behavior.

Financial markets and corresponding networks may have a huge amount of assets (nodes) and inter- 
connections (edges). It is useful to simplify initial networks by filtering the noises. Some of the approaches employed in filtering of financial networks are based on hierarchical tree (HT) [3, 4], planar maximally filtered graphs (PMFG) [5], asset graph (AG) [6], and partial correlation network [7, 8, 9]. Another successful method of financial network simplification was developed by Mantegna [3] and applied in [10]. The approach is based on the minimum spanning tree method for examining of hierarchical structures of financial networks.

In recent years there has been an increased interest in the application and development of an approach based on market graphs. These papers include empirical studies based on real market data and explore various structural properties and attributes of market graphs, such as maximum clicks, maximum independent sets, degree distribution [11, 12,13,14], clustering, Pearson correlations [15], market graphs of the US market [16], the complexity of the market graph [17]. The concept of a market graph was first considered in [18], in which a market network is defined as a full weighted graph, where nodes represent assets and arc weights reflect similarities between asset behavior (which can be measured, for example, using correlation). In article [18], the edge between two vertices is inserted into the market graph, if the corresponding value of the correlation coefficient is above a given threshold. The articles [19, 20, 21, 12, 22] studied the distinctive features of individual financial markets. Market graphs with similarity measures other than correlation were studied in [19, 23, 24, 25, 8, 26].

In this paper, we use some methods of complex network theory to study Russian financial market network. Complex network analysis and SNA methods allow us to investigate the structure of Russian financial market network that are based on the correlation relationship between the components of the system (assets).

Network analysis methods have proved to be successful in studying various individual major financial markets such as US market [27, 28, 29], Germany [30, 4], EU [31, 32, 33], Italian [34, 35]. Some research papers have been investigate developing markets such as China [29, 36, 12, 37], Brazil [38, 39], Korea [40], Russia [21], and Mexico [41, 42]. Moreover, some papers employed network approach to examine global markets [43, 44].

It turned out that the network analysis is capable to examine the importance of companies using different centrality measures $[45,45]$ and to analyze the systemic risks and financial market stability based on the topological properties of networks [46, 47, 48]. Systemic risk contagion has been analyzed in $[49,50]$ to find major influencers based on banking behaviors in the global environment. The paper [51] studies European markets and shows that EU markets are exposed to systemic risks. It should be noted that systemic risk analysis based on network approach may provide useful implications for market regulations.

The paper [29] employs various methods of network analysis to investigate financial networks of both in China and the United States and to study how the two markets behave differently. In this paper, we will present a similar analysis of Russian stock market. One of the main research questions of this paper is to find how the US and China stock markets differ in the structures and topological properties from Russian stock market using

- market graph approach proposed in $[18,11]$,

- hierarchical tree (HT) method [3],

- minimum spanning tree (MST) approach [3].

In this paper we compare topological properties of the networks constructed for the US and China stock markets in [29] with the properties of corresponding networks constructed for the Russian stock market using a dataset spanning over nine years.

The paper is organized as follows. First, Section 2 describes the data and method used to construct networks. Section 3 presents the market network properties using market graph approach proposed in $[18,11]$. Section 4 examines the hierarchical structures of the Russian stock network using HT, and MST approaches and compares them with findings of the paper [29]. Finally, conclusions are presented in Section 5.

\section{Data and Methodology}

\subsection{Data Description}

Russia has a developing economy which is the sixth among countries in the world in terms of GDP (PPP). The RTS index and the Moscow Stock Exchange index are the main indicators of the Russian stock market. The difference between them is that the RTS is calculated in dollars, and the MICEX in rubles. Historical data of daily prices for each company were taken from open sources on Yahoo Finance, for the period from 10/01/2012 to 09/04/2019 (1664 trading days). Similarly, data on the MICEX index were taken 
from the Moscow Stock Exchange for the same period. A total of 194 companies are considered, among which 32 companies are part of the MICEX index.

The descriptive statistics for log returns of the IMOEX index over the period between 10/01/2012 and 09/04/2019 are presented in Table 2.2. The result of $\operatorname{Garch}(1,1)$ modelling for log returns of the IMOEX index over the period between 10/01/2012 and 09/04/2019 is presented in Fig. 1.

Some companies (CBOM, DSKY, FIVE, LNTA, MOEX, POLY, RNFT, RUAL, SFIN) that are part of the MICEX index were not included in our dataset. This is due to the fact that they have a very short trading history (only 2-3 years), which is insufficient to build a relevant model. We also did not include companies that had more than 250 missed trading days in a row in our dataset. Thus, only 194 issuers remained in our dataset from the initial list of 278 issuers traded on the Moscow stock exchange. The YNDX company has started to be traded on the Moscow stock exchange from $02 / 06 / 2014$, but it has been traded on the NASDAQ from 02/05/2011, from which the missing data were taken. After cleaning and the selection process, we include 194 stocks traded on IMOEX in our dataset, as shown in Table 1. We place these 194 stocks among all ten industry sectors. Table 2.2 presents basic information about datasets.

\subsection{Market Network Construction}

To calculate the correlation $\rho_{i j}$ for a pair of stocks, it is necessary to use time series of prices (Adj Close) $P_{i}(t)$ for each company $s_{i}$ at the time point $t$. To smooth the oscillations we use the log returns $Y_{i}(t)$ of the company $s_{i}$ in the time period $[t-\Delta t, t]$ defined by

$$
Y_{i}(t)=\ln P_{i}(t)-\ln P_{i}(t-\Delta t),
$$

where $\Delta t=1$ for daily prices. Then we find the Pearson correlation coefficient for each pair of companies $s_{i}$ and $s_{j}$ as follows

$$
\rho_{i j}=\frac{\left\langle Y_{i} Y_{j}\right\rangle-\left\langle Y_{i}\right\rangle\left\langle Y_{j}\right\rangle}{\sqrt{\left(\left\langle Y_{i}^{2}\right\rangle-\left\langle Y_{i}\right\rangle^{2}\right)\left(\left\langle Y_{j}^{2}\right\rangle-\left\langle Y_{j}\right\rangle^{2}\right)}}
$$

where $\langle\cdot\rangle$ denotes the average value.

Based on the values of the Pearson correlation coefficient for each pair of companies we can construct the $N \times N=194 \times 194$ distance matrix using the equation

$$
d_{i j}=\sqrt{2\left(1-\rho_{i j}\right)} .
$$

\begin{tabular}{lc}
\hline Sector & Stocks \\
\hline Basic Materials & 36 \\
Consumer Cyclicals & 11 \\
Consumer Non-Cyclicals & 9 \\
Energy & 22 \\
Financials & 16 \\
Healthcare & 3 \\
Industrials & 24 \\
Technology & 3 \\
Telecommunications Services & 11 \\
Utilities & 59 \\
\hline
\end{tabular}

Table 1: 194 component stocks of IMOEX are included in our dataset. In this table, we list sector name, and numbers of stocks for each industry sector of these 194 stocks. All 10 industry sectors are represented

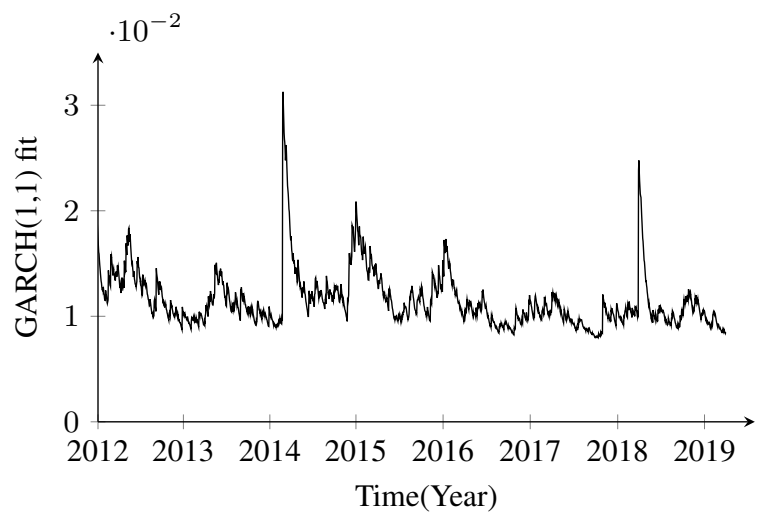

Figure 1: $\operatorname{GARCH}(1,1)$ time series of the volatility of the IMOEX index daily log returns

\section{Basic network properties}

In this section, we examine the topological properties of the IMOEX stock network using the dynamic approach applied in [29]. We take the sliding window size $L=250$ to meet the requirement of $L / N>1$, where the number of stocks is $N=194$. In total, there are 1413 windows for IMOEX. We calculated the log returns for all IMOEX stocks by using Eq. (1) and then we found the Pearson correlation coefficient matrix for each window over the period between 10/01/2012 and 09/04/2019 using Eq. (2).

We compare the results obtained for the Russian stock market with the results obtained in the paper [29] for the Chinese and US stock markets. The dataset S\&P468 has been described in [29] and cosnsists of 468 component stocks of S\&P500. The 


\begin{tabular}{ccccccc}
\hline Dataset & Stocks & Days & $\langle Y\rangle$ & $\sigma_{Y}$ & $\left\langle Y_{\min }\right\rangle$ & $\left\langle Y_{\max }\right\rangle$ \\
\hline IMOEX & 194 & 1664 & $2.66 \mathrm{e}-4$ & 0.0125 & -0.1141 & 0.0551 \\
CSII63 & 163 & 2149 & $1.48 \mathrm{e}-04$ & 0.0340 & -0.4832 & 0.1002 \\
S\&P468 & 468 & 2228 & $1.47 \mathrm{e}-04$ & 0.0252 & -0.3413 & 0.2160 \\
\hline
\end{tabular}

Table 2: Basic information of IMOEX datasets including the number of stocks, the number of trading days, the average $\log$ returns $\langle r\rangle$, and the standard deviation $\sigma_{r}$ of the log returns is presented

dataset CSI163 has been described in [29] and includes of 163 component stocks of CSI300.

Thus, for IMOEX we obtain a network with 194 vertices. Using Eq. (3) we calculate the distance matrices for each window. It should be noted that all negative correlation values are transformed into positive distances. All vertices in the network are fixed and correspond to stocks. However, the edges vary in each sliding window as the distances change. Then we average all the distance matrices over all sliding windows of the study periods. The statistical properties of the IMOEX stock network are examined in this section.

The maximum number of edges in average distance matrix is equal to $N(N-1) / 2=18,721$ and it is a huge number. We can simplify the network by filtering edges with small weights. In this section the market graph is constructed based on threshold approach: for a fixed threshold value of $\theta$ we delete those edges whose weight are bigger than $\theta$ and preserve the remaining edges. In other words, we filter the network edges as follows:

$$
e_{i j}=\left\{\begin{array}{l}
1, d_{i j}<\theta, \\
0, d_{i j} \geq \theta
\end{array}\right.
$$

Table 3 presents the basic properties of the IMOEX network for different values of $\theta$ taken from 0.5 to 1.5 . It can be seen that the number of edges is zero if the threshold value is $\theta=0.8$, while the graph becomes complete at $\theta=1.5$. The figure 2 shows edge densities for different thresholds from 0.5 to 1.5 . In the interval from 0.5 to 1.1 , network densities are close to 0 , which means that all edges are filtered. In the range from 1.43 to 1.5 , the densities are close to 1, i.e. all edges are present at the network. For charts based on Chinese and American stock markets [29] we see that the two curves have a similar shape with a slopes that lie between 0.6 and 1 . It should be noted that the transformation interval for the IMOEX network is from 1.1 to 1.43 , which is atypical for stock networks. As a rule, stock networks show the transformation interval from 0.6 to 1 . The study shows that Russian stock network has a different form of trans- forming interval than the USA and China . This is due to much lower correlations between assets traded in the Russian stock market, which could be explained by e.g. low liquidity of most stocks in IMOEX. Moreover, it can be easily verified that even highest liquidity shares provide low correlations. Network density for a graph constructed using these stocks maintains similar dependency from $\theta$ as for the whole IMOEX network.

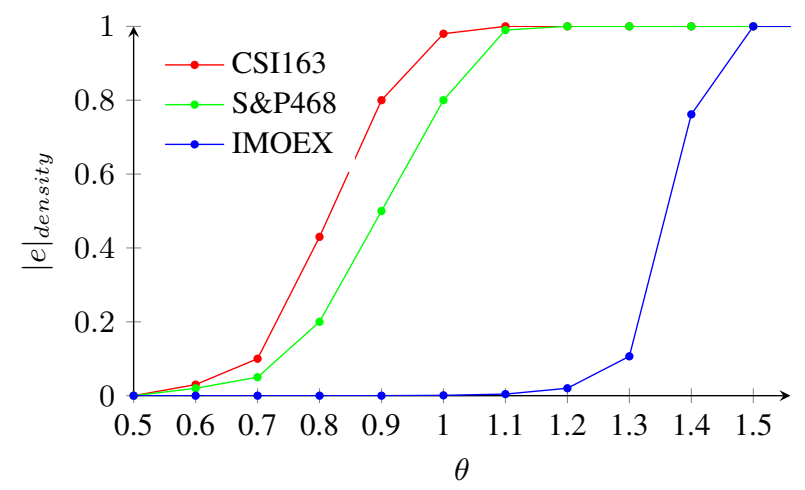

Figure 2: Edge densities of CSI163, S\&P468 and IMOEX for different thresholds $\theta$ from 0.5 to 1.5 . It shows that the densities for IMOEX increase sharply from 0 to 1 in the interval of $\theta$ from 1.05 to 1.45 .

\subsection{Degree distribution}

We investigate the degree distributions for the IMOEX networks filtered with different values of $\theta$. The degree distributions are noisy for both small values of $\theta$ and large ones. For a sufficiently small interval $\theta=$ $(1.15 ; 1.2)$, the degree distributions follow closely to the power law distribution. After processing the data, we build the degree distribution with the typical power law shape in Fig. 3. The log-binning procedure was used to build the regression and calculate the power law exponent. The value of the power law exponent is $\gamma=1.14$ with $R^{2}=0.85$. Since the use of linear approximation (linear-binning) significantly underestimates the exponent $\gamma$. A negative tilt angle indicates that the network is scale-free, in which a small part of 


\begin{tabular}{cccccccc}
\hline$\theta$ & $|e|_{\max }$ & $|e|$ & $|e|_{\text {density }}$ & $\langle d\rangle$ & $\left\langle d_{i j}\right\rangle$ & $d_{i j}^{\max }$ & $\sigma$ \\
\hline 0.5 & 18721 & 0 & 0.0000 & 0.0000 & 0.0000 & 0.0000 & 0.0000 \\
0.6 & 18721 & 1 & 0.0001 & 0.0103 & 0.5458 & 0.5458 & 0.0000 \\
0.7 & 18721 & 2 & 0.0001 & 0.0206 & 0.5848 & 0.6238 & 0.0552 \\
0.8 & 18721 & 2 & 0.0001 & 0.0206 & 0.5848 & 0.6238 & 0.0552 \\
0.9 & 18721 & 4 & 0.0002 & 0.0412 & 0.7201 & 0.8969 & 0.1631 \\
1 & 18721 & 17 & 0.0009 & 0.1753 & 0.8957 & 0.9994 & 0.1263 \\
1.1 & 18721 & 80 & 0.0043 & 0.8247 & 1.0235 & 1.0992 & 0.0915 \\
1.2 & 18721 & 376 & 0.0201 & 3.8763 & 1.1351 & 1.1999 & 0.0756 \\
1.3 & 18721 & 1996 & 0.1066 & 20.5773 & 1.2393 & 1.2998 & 0.0648 \\
1.4 & 18721 & 14260 & 0.7617 & 147.0103 & 1.3477 & 1.4000 & 0.0551 \\
1.5 & 18721 & 18721 & 1.0000 & 193.0000 & 1.3638 & 1.4748 & 0.0563 \\
\hline
\end{tabular}

Table 3: The max possible number of edges $|e|_{\max }$ for $N=194$ vertices, the existing number of edges $|e|$, the edge density $|e|_{\text {density }}$, the average degree $\langle d\rangle$, the average distance $\left\langle d_{i j}\right\rangle$, the maximum distance $d_{i j}^{\max }$ are presented for different $\theta$ from 0.5 to 1.5 in a step of 0.1 , the standard deviation $\sigma$

the vertices has greater degrees, and most of the vertices have smaller degrees.

Fig. 3 shows that the degree distributions of IMOEX (with $\theta=1.2$ ), CSI163 (with $\theta=0.68$ ) and S\&P468 (with $\theta=0.75$ ) networks follow the power law in the form of $p_{k} \sim k^{-\gamma}$ with $\gamma=1.14, \gamma=$ 0.9935 and $\gamma=1.2323$ respectively. It means that the networks are scale free in which a small portion of vertices have larger degrees, while a large portion of vertices have small degrees. At the first sight, the IMOEX network has the same scale-free behaviour as the CSI163 and S\&P468 networks. However, if we take the same level of $\theta$ as it was taken for the CSI163 and S\&P468 networks (i.e. $\theta=0.65 \pm 0.1$ ) then the IMOEX network will be very sparse and almost all vertices will be isolated.

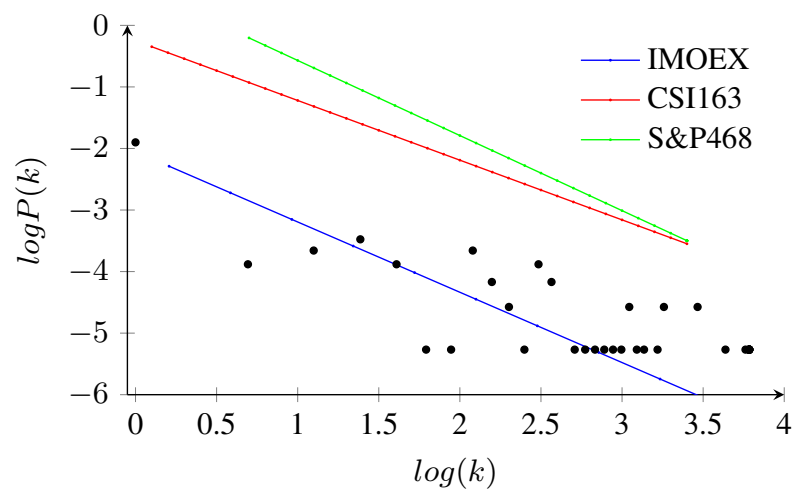

Figure 3: Log-Log degree distributions of IMOEX, CSI163 and S\&P468 networks. By using different $\theta$, we can filter out edges with larger distances. This figure shows the $\log$-Log degree distribution of IMOEX (with $\theta=1.2$ ), CSI163 (with $\theta=0.68$ ) and S\&P468 (with $\theta=0.75$ ) networks.

\subsection{Average clustering coefficient}

The average clustering coefficient (ACC) is the average of all clustering coefficients over all vertices. The clustering coefficient means transitivity for a single vertex, while the average clustering coefficient is an indicator of the transitivity and density for the entire network. Fig. 4 presents the average clustering coefficient for the IMOEX network and its comparison with a random network. Compared to the random network of the same size, the ACC for the IMOEX network is significantly larger than that of the random network. It shows that the IMOEX network is well connected with better transitivity. For comparison, Fig. 4 presents the average clustering coefficients for China and the USA networks. Fig. 4 shows that the IMOEX network does not behave in the similar way: ACC values are always lesser than for CSI163 and S\&P468 networks, i.e. the transitivity and density for the IMOEX network are much lower than for China and the USA networks.

\subsection{Average path length}

Fig. 5 shows the average path length for the IMOEX network compared to a random network of the same size. It can be seen that the IMOEX network is significantly different from the random network. For comparison, Fig. 5 also shows the average clustering coefficients for China and the USA networks. Fig. 5 indicates that the IMOEX network behaves differently: the average path length has a peak at $\theta=1.1$ while the CSI163 and S\&P468 networks have peaks at $\theta=0.6$ and 0.65 respectively. Moreover, the average path length for the IMOEX network is much bigger than for the CSI163 and S\&P468 networks for $\theta>1$. Thus, the China and the USA networks are more con- 


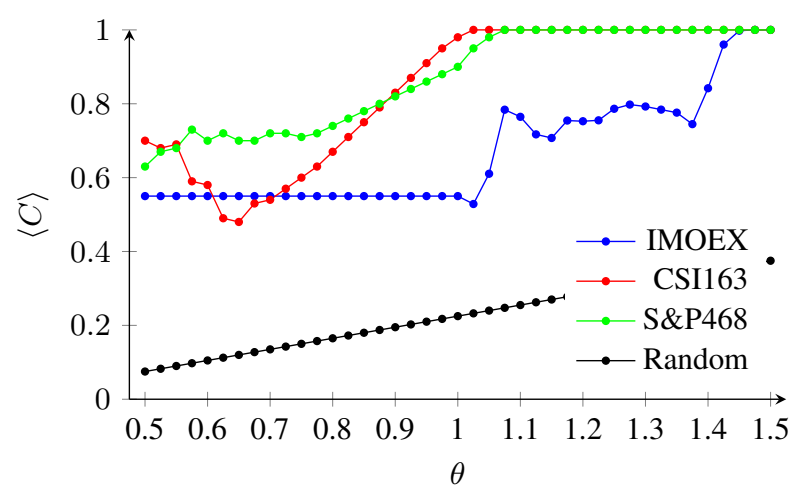

Figure 4: Average clustering coefficient $\langle C\rangle$ of IMOEX, CSI163 and S\&P468 for different thresholds $\theta$.

nected in the sense that economic shocks and important financial and economic news can transfer along networks much quicker.

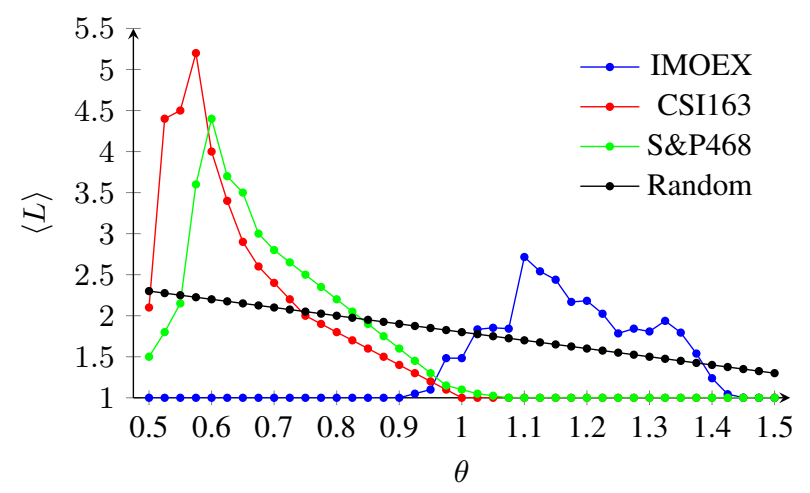

Figure 5: Average path lengths $\langle L\rangle$ for IMOEX, CSI163 and S\&P468 under diferent $\theta$ compared with values for random networks of size 194 .

\subsection{Betweenness centrality}

Betweenness of a vertex is defined as the number of shortest paths passing through it. Thus, this indicator demonstrates the contribution of each vertex to global connectivity. Averaging over individual betweenness coefficients over all vertices, we can compare the betweenness values for any two vertices. Fig. 6 shows that the peak of the average betweenness for the network is achieved at the threshold value $\theta=1.35$. It can be seen (Fig. 5) that the CSI163 and S\&P468 networks have peaks at $\theta=0.62$ and 0.81 respectively. Moreover, the maximum values of the average betweenness for the IMOEX network are much lower than for the CSI163 and S\&P468 networks. Thus, the China and the USA networks have a greater number of nodes with higher betweenness centrality that have more control over the network (in comparison with the IMOEX network).

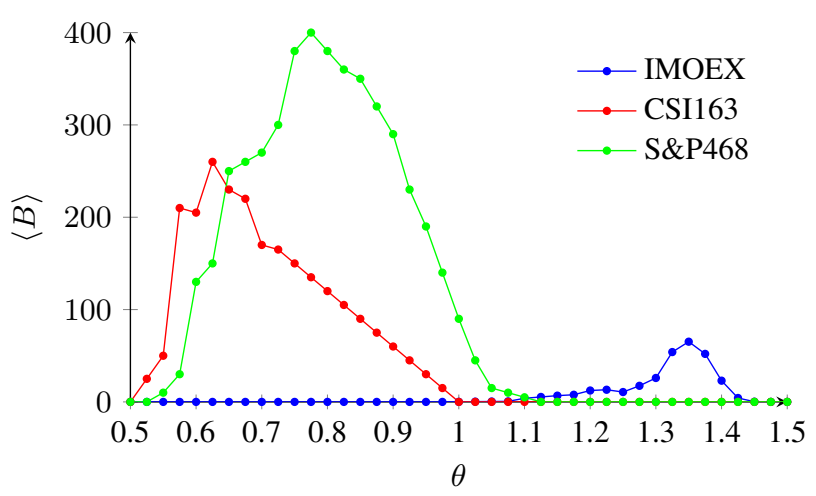

Figure 6: Average betweenness of IMOEX, CSI163 and S\&P468

We visualize the stock network of IMOEX with different values of $\theta=1.1,1.2$ in Fig. 7. The figures show that the network can be greatly simplified by using small values of $\theta$. Table 3 indicates that the edge density for the IMOEX network increases dramatically from $\theta=1.1$ to $\theta=1.4$.

\subsection{Components}

A component of a network is defined as a subnetwork with connected vertices. If a network has $N$ vertices, then the size of component can vary from 1 (for an isolated node) to the maximum value $N$ (ifr all vertices are connected). The number of components, the max component size, and the average component size are plotted in Fig. 8, 9 and 10 for the IMOEX, CSI163 and S\&P468 networks filtered with different threshold values. The figure shows transitions of the networks from a large number of small isolated components into a connected giant network. At the same time, for the average component size there is a sharp transition to the giant component at $\theta \approx 1.35$.

To examine how industry sectors are linked in the IMOEX network, we list the properties of the network with $\theta=1.4$ in Table 3.5. The table shows that the Russian industry sectors has approximately the same average degree as well as the average clustering coefficient. However, the values of average betweenness coefficient differ for different sectors assuredly. It should be noted that the same results were obtained for the CSI163 and S\&P468 networks in [29]. 


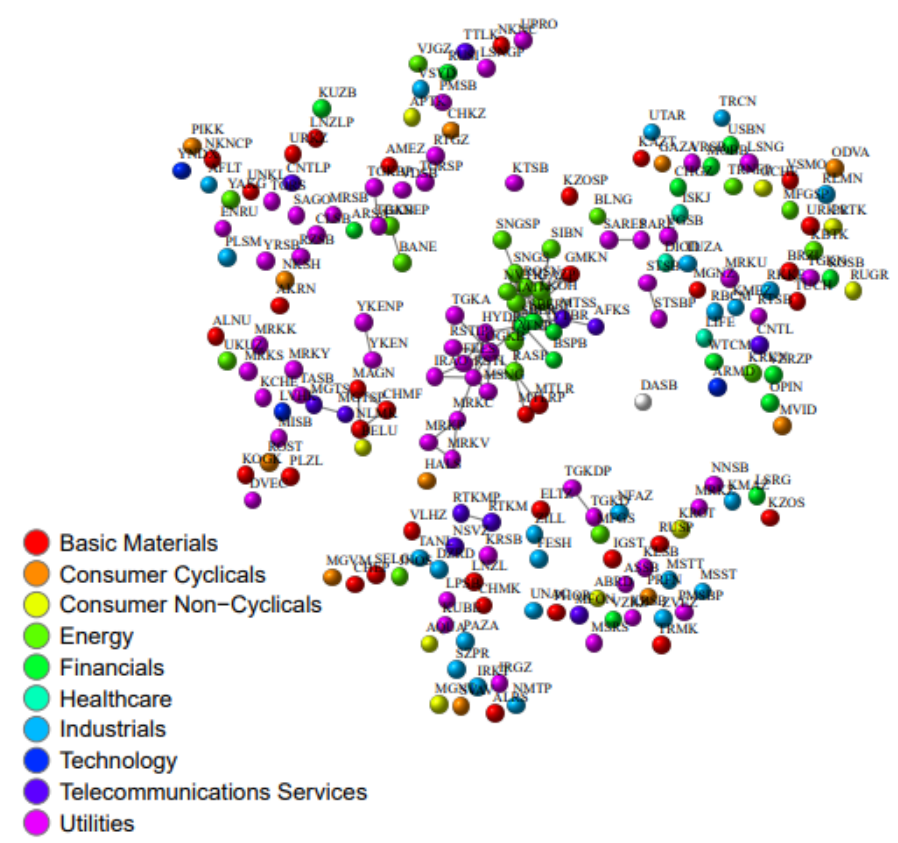

(a) IMOEX network $\theta=1.1$

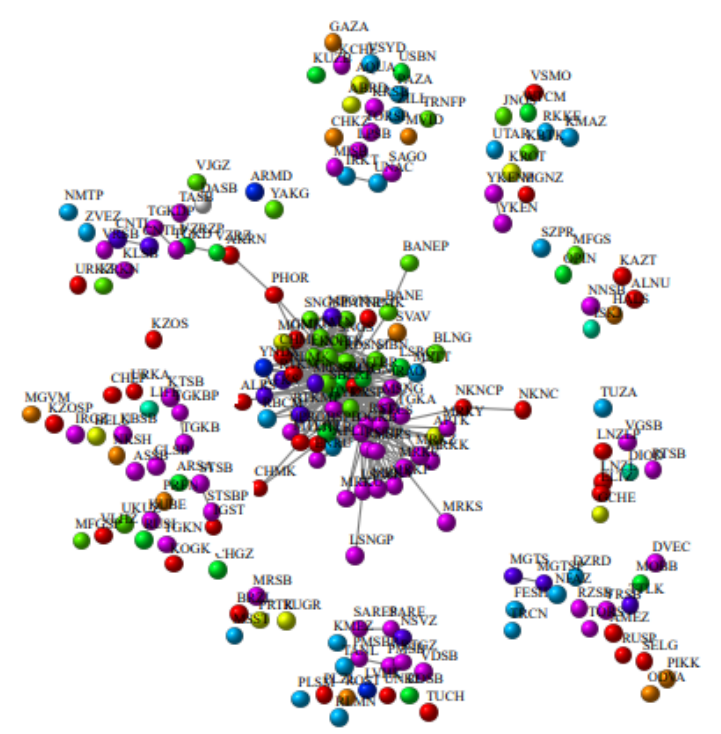

(b) IMOEX network $\theta=1.2$

Figure 7: IMOEX networks with different $\theta$ of 1.1 (a), 1.2 (b)

\begin{tabular}{ccccc}
\hline Sector & Number & $\langle d\rangle$ & $\langle C\rangle$ & $\langle B\rangle$ \\
\hline Basic Materials & 36 & 150.9722 & 0.8516 & 11.5556 \\
Consumer Cyclicals & 11 & 141.0909 & 0.8472 & 7.9091 \\
Consumer Non-Cyclicals & 9 & 154.5556 & 0.8539 & 3.0000 \\
Energy & 22 & 153.6818 & 0.8366 & 40.1364 \\
Financials & 16 & 147.7500 & 0.8342 & 53.5000 \\
Healthcare & 3 & 159.000 & 0.8618 & 8.3333 \\
Industrials & 24 & 132.3333 & 0.8424 & 10.2500 \\
Technology & 3 & 155.0000 & 0.8559 & 0.6667 \\
Telecommunications Services & 11 & 152.2727 & 0.8449 & 29.8182 \\
Utilities & 59 & 145.8305 & 0.8350 & 26.9661 \\
\hline
\end{tabular}

Table 4: In this table, we list the sector name and the numbers of stocks, the average degree $\langle d\rangle$, the average clustering coefficient $\langle C\rangle$, and the average betweenness coefficient $\langle B\rangle$ for each industry sector of these 194 stocks. The values are calculated from the IMOEX network with $\theta=1.4$.

Table 3.5 presents the top 10 stocks of IMOEX network with the biggest values of degree $d_{i}$ and betweenness $b_{i}$ arranged in descending order in the top part and the bottom part, respectively. The stock code, company name, industry name, and values of degree $d_{i}$, clustering coefficient $c_{i}$ and betweenness $b_{i}$ are presented. SBERBANK, which is a leading financial company in Russia, has the largest degree of 136 and JSC "OGK-2" is Russia's largest heat generating company, has the largest betweenness coefficient of 1551 .

\section{Hierarchical Structure of Stock Networks}

Graphs constructed by certain rules may reveal its properties. In this section we create and study hierarchical trees and minimal spanning tree to extract valuable fragments from the graph based on the average distance matrix, which we construct by averaging sliding windows over the study period.

\subsection{Hierarchical Tree}

Clustering algorithms can be used on average distance matrix to group stocks which prices correlate and to 


\begin{tabular}{lllcccc}
\hline Ticker & Name & Industry & $d_{i} \downarrow$ & $c_{i}$ & $b_{i}$ & Cap \\
\hline SBER & SBERBANK & Financials & 136 & 0.5082 & 1407 & $4.92 \cdot 10^{6}$ \\
OGKB & OGK-2 & Utilities & 136 & 0.5031 & 1551 & $4.25 \cdot 10^{4}$ \\
RASP & RASPADSKAYA & Energy & 132 & 0.5191 & 1218 & $9.60 \cdot 10^{4}$ \\
RSTI & ROSSETI & Utilities & 131 & 0.5400 & 719 & $2.16 \cdot 10^{5}$ \\
SBERP & SBERBANK OF RUSSIA & Financials & 128 & 0.5519 & 556 & $4.90 \cdot 10^{6}$ \\
FEES & FSK EES & Utilities & 128 & 0.5560 & 249 & $2.08 \cdot 10^{5}$ \\
VTBR & VTB & Financials & 127 & 0.5668 & 131 & $2.29 \cdot 10^{2}$ \\
MSNG & MOSENERGO & Utilities & 125 & 0.5735 & 144 & $8.96 \cdot 10^{4}$ \\
BSPB & BANK ST PETERSBURG & Financials & 125 & 0.5472 & 499 & $2.80 \cdot 10^{4}$ \\
RSTIP & ROSSETI & Utilities & 122 & 0.5780 & 215 & $2.14 \cdot 10^{5}$ \\
\hline Ticker & Name & Industry & $d_{i}$ & $c_{i}$ & $b_{i} \downarrow$ & Cap \\
\hline OGKB & OGK-2 & Utilities & 136 & 0.5031 & 1551 & $4.25 \cdot 10^{4}$ \\
SBER & SBERBANK & Financials & 136 & 0.5082 & 1407 & $4.92 \cdot 10^{6}$ \\
RASP & RASPADSKAYA & Energy & 132 & 0.5191 & 1218 & $9.60 \cdot 10^{4}$ \\
RSTI & ROSSETI & Utilities & 131 & 0.54 & 719 & $2.16 \cdot 10^{5}$ \\
MRKC & IDGC CENTRE & Utilities & 114 & 0.6167 & 700 & $1.28 \cdot 10^{4}$ \\
SBERP & SBERBANK OF RUSSIA & Financials & 128 & 0.5519 & 556 & $4.90 \cdot 10^{6}$ \\
GAZP & GAZPROM & Energy & 119 & 0.611 & 531 & $3.62 \cdot 10^{6}$ \\
BSPB & BANK ST PETERSBURG & Financials & 125 & 0.5472 & 499 & $2.80 \cdot 10^{4}$ \\
KBSB & TNS ENERGO KUBAN & Utilities & 4 & 0.3333 & 352 & $2.36 \cdot 10^{3}$ \\
LSNG & LENENERGO & Utilities & 103 & 0.665 & 323 & $5.80 \cdot 10^{4}$ \\
\hline
\end{tabular}

Table 5: Top stocks with highest values of degree $d_{i}$, clustering coefficient $c_{i}$ and betweenness $b_{i}$ ranked in descending order of $d_{i}$ and $b_{i}$ for IMOEX network with the $\theta=1.35$. Stock tickers in bold indicate the stocks appear at both top 10 stocks. Cap denotes capitalization in millions of RUB.

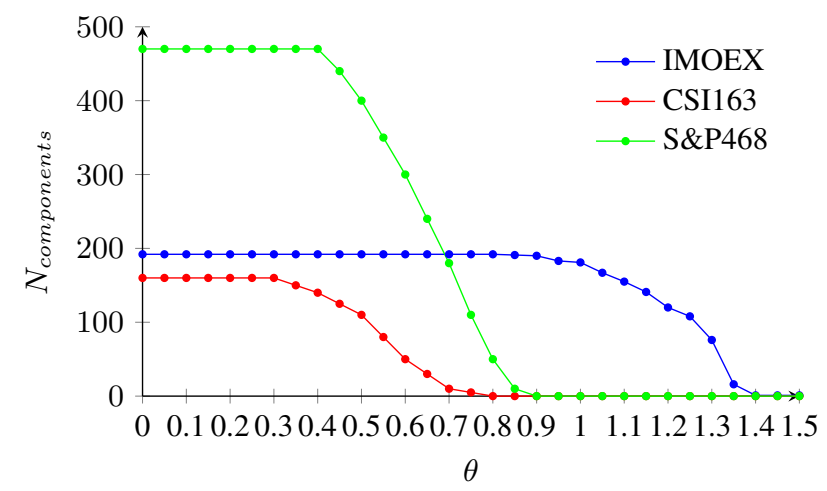

Figure 8: The number of components for IMOEX, CSI163 and S\&P468 networks.

separate rest. Multiple definitions for distance between clusters can be used. Since clustering algorithm depends on how distance value is calculated, we used all four definitions. The distance $d_{a, b}$ between two clusters $c_{a}$ and $c_{b}$ is defined as minimum, maximum and average for all pairs $\left(o_{a}, o_{b}\right)$ from $c_{a}$ and $c_{b}$ respectively or as distance between their centroids: $d_{a, b}=\max \left(d_{o_{a}^{i}}, d_{o_{b}^{j}}\right), d_{a, b}=\min \left(d_{o_{a}^{i}}, d_{o_{b}^{j}}\right)$, $d_{a, b}=\frac{1}{N_{m} N n} \sum_{i} \sum_{j} d_{o_{a}^{i}, o_{b}^{j}}, d_{a, b}=d\left(\overline{o_{a}}, \overline{o_{b}}\right)$. We create four hierarchical trees each using its own nor-

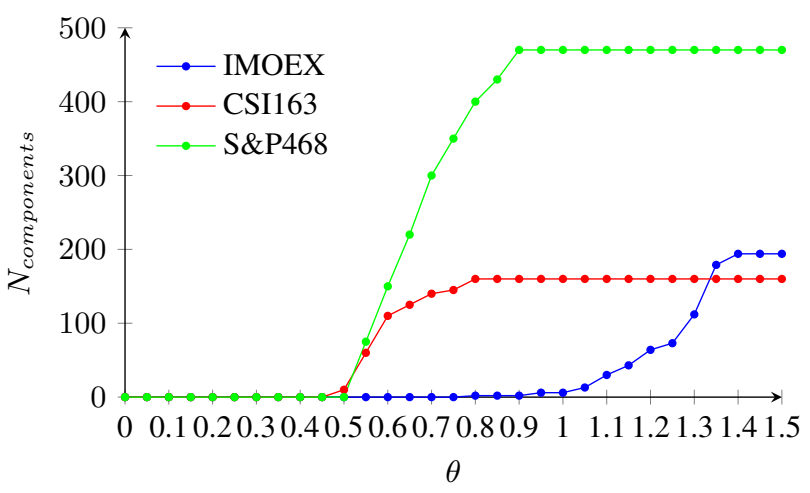

Figure 9: The max component size for the IMOEX, CSI163 and S\&P468 networks.

malised distance definition (Fig. 11). Dendogram function from Scipy package is used to draw them on a plot from Matplotlib package. In the trees, each leaf node indicates the stock of a particular company with its ticket as a label. The height of where two stocks connect shows how they correlate. The lower the branches of similar clusters or stocks merge the more positive correlation there is between them. We set color threshold of $0.7 \times \max \left(d^{*}\right)$ to highlight clusters below this value. The companies in same colored branches are very likely to be from the same economy 


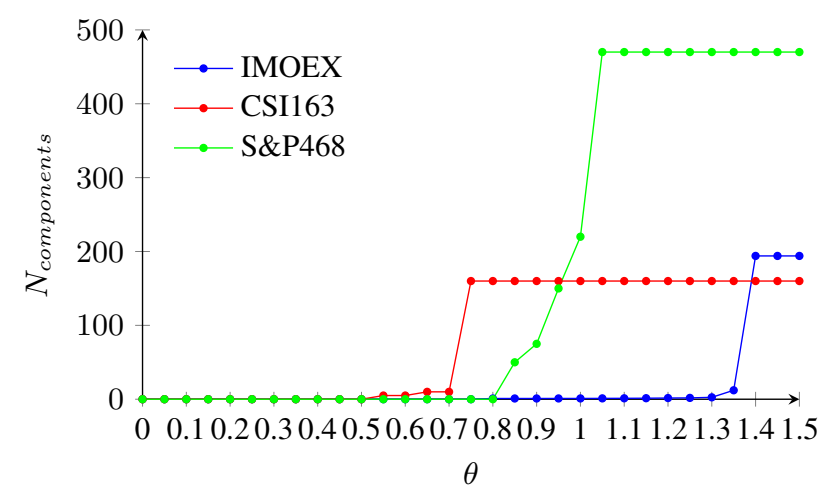

Figure 10: The average component size for the IMOEX, CSI163 and S\&P468 networks.

sectors which means that hierarchical trees can show economy sectors from price correlation matrix alone.

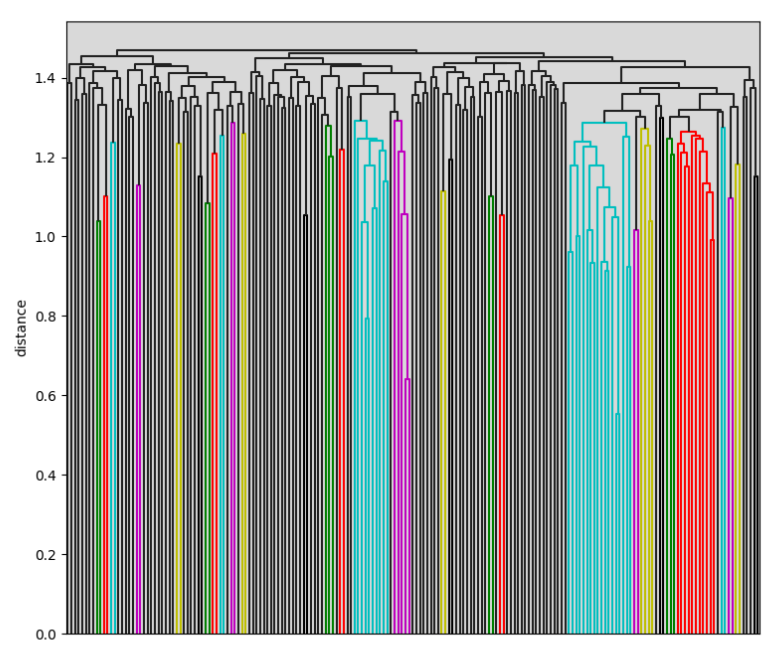

(a)

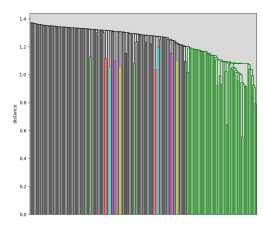

(b)

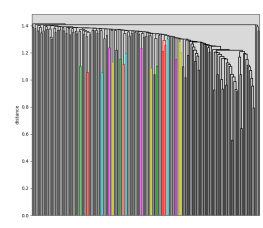

(c)

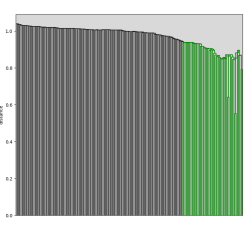

(d)
Figure 11: Hierarchical clustering trees based on average distance for stocks in IMOEX using: (a) complete-linkage, (b) single-linkage, (c) average linkage, (d) centroid linkage

The maximum-based linkage criterion showed better clustering results if we compare four trees generated by the hierarchical clustering algorithms.

\subsection{Minimum Spanning Tree}

IMOEX network, based on average distance matrix, can be simplified by extracting edges with minimal distance while maintaining graph connectivity. Connected graph with $N$ vertices can be converted into the graph with $N-1$ edges without loops with minimum total length of edges or minimum spanning tree (MST). This graph is called minimum spanning tree. It brings huge advantages to the study of networks of stocks by reducing noises and simplifying computations. We use Python Scipy minimum spanning tree algorithm on an average distance matrix. Networkx python package is used to get its graphic representation on a Matplotlib plot as shown in Fig. 12.

After the edge filtering process, some nodes are still well-connected, and they may be considered the key stocks in the market. The most connected stocks are from utilities, financials and energy sectors. These results let us extract the most influential companies from the given data. It should be noted that Markowitz optimal portfolio consists of stocks corresponding to leaf nodes of MST. Therefore, we can use MST approach for portfolio optimisation.

Top 10 stocks are shown in the table 4.2. As expected, financials, energy and utilities sectors are dominant in the Russian stock market which is different from the US and Chinese stock markets. Further studies of minimum spanning tree over time may reveal important changes in the core stocks and sectors of the market. Moreover, the result of the algorithm application differs between study periods and this is helpful when studying network topological dynamics.

\subsection{Hierarchical tree for a pooled market graph (IMOEX and S\&P468)}

The obtained results indicate a significant difference in the density of correlation between stock returns and the characteristics of market graphs constructed for the financial markets of the US, China and the Russian Federation.

Such differences can be explained by the relatively less developed financial market of the Russian Federation as a whole, and industry affiliation of companies, greater uncertainty and a high share of risks specific to each of the companies, etc. It is known that a significant share in the total capitalization of the financial market of the Russian Federation falls 


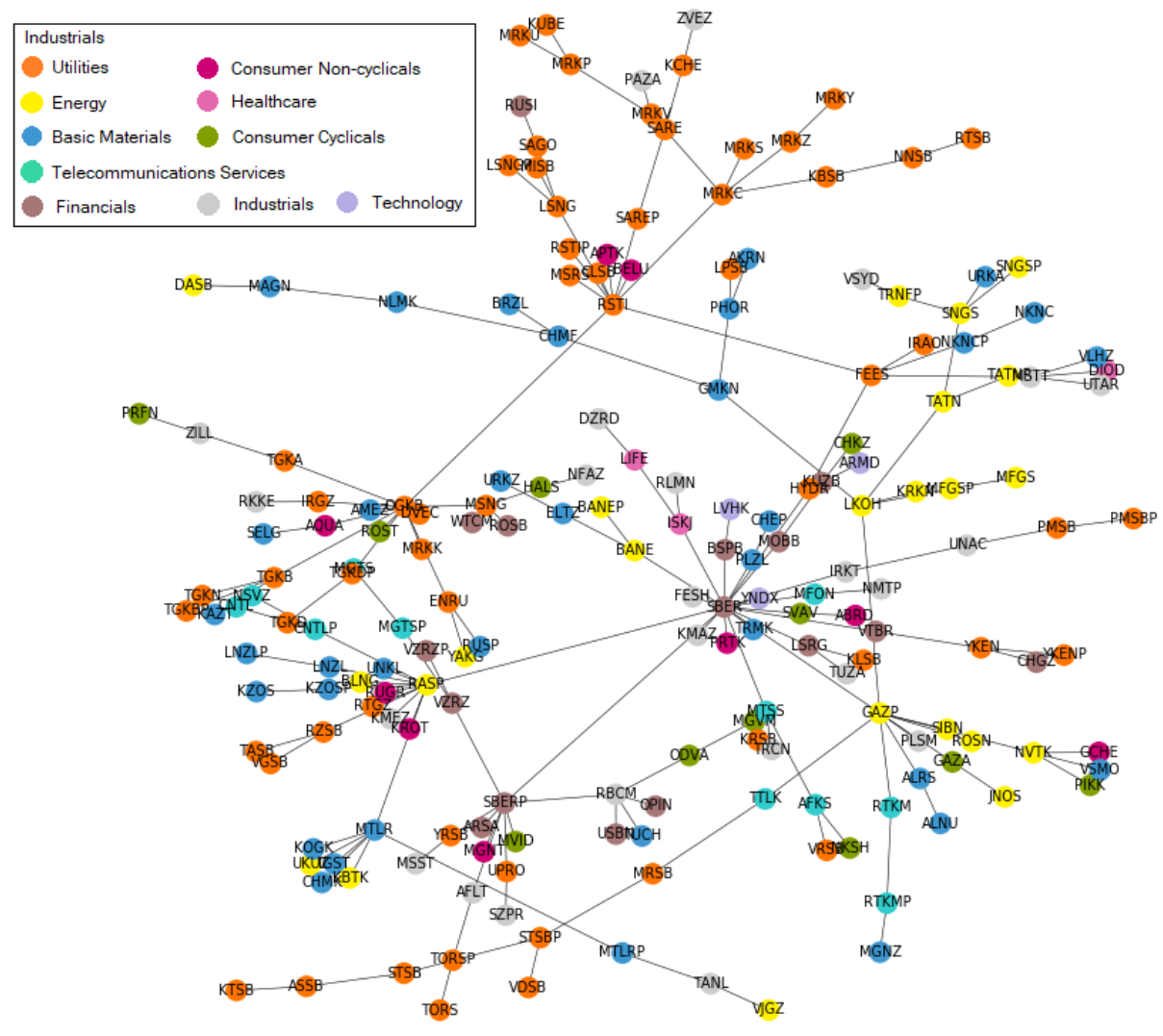

Figure 12: Minimal spanning tree for IMOEX network

\begin{tabular}{cccc}
\hline Degree & Ticket & Name & Industry \\
\hline 19 & SBER & SBERBANK & Financials \\
12 & RASP & RASPADSKAYA & Energy \\
11 & OGKP & OGK-2 & Utilities \\
10 & RSTI & ROSSETI & Utilities \\
9 & SBERP & SBERBANK & Financials \\
8 & GAZP & GAZPROM & Energy \\
7 & MTLR & MECHEL & Basic Materials \\
5 & MRKC & IDGC CENTRE & Utilities \\
5 & FEES & FSK EES & Utilities \\
5 & LKOH & LUKOIL & Energy \\
\hline
\end{tabular}

Table 6: In this table 10 most significant stocks are shown. The most important stocks are from three industries: utilities, financials and energy

on the shares of companies focused on the export of raw materials. It is interesting to check whether industry or country is more important for the correlation dependence. To do this, we apply the hierarchical tree method for a pooled market graph (IMOEX and S\&P468). If industry factors would be more important, then Russian stocks should be placed to clusters according to their industry affiliation. If cross-country differences are more important, then clusters should be placed to clusters according to their country affili- ation.

The results of applying the hierarchical classification method are presented in the Fig. 13. All Russian companies fall into one cluster (in the figure it is marked in blue at the left side of the dendrogram). S\&P500 companies are located in other clusters, which are well explained by their industry affiliation. 


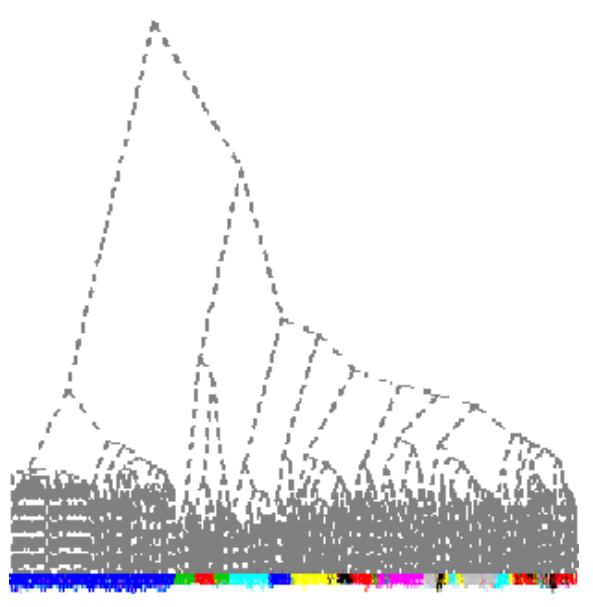

Figure 13: Hierarchical tree for a pooled market graph (IMOEX and S\&P468)

\section{Conclusion}

In this paper, using the daily close prices we calculated the correlation matrices for 194 assets trading on the Russian stock exchange during the study period from 2012 to 2019 for all sliding windows. Based on these correlation matrices we constructed the market networks with stocks as the nodes and correlation relationships as the edges. Our goal was to study the networks properties using the complex network theory from data science perspective. We employed such network filtering methods as threshold, hierarchical tree, minimum spanning tree for simplification of the networks. Then we examine the network properties for the filtered networks. We compare topological properties of the networks constructed for the US and China stock markets with the properties of corresponding networks constructed for the Russian stock market using a dataset spanning over eight years. It turns out that the IMOEX network share similar topological properties in some case, but it also differ from the CSI163 and S\&P468 networks in many aspects. The study shows that the topological properties of the Russian stock network has a different from the USA and China dependence on $\theta$, which is the consequence of a much lower correlations between assets traded in the Russian stock market. One the one hand, it may be explained by low liquidity of most stocks traded in IMOEX. On the other hand, even shares with high liquidity have low correlations.

Acknowledgements: This work was supported by the Russian Science Foundation, project 19-18-00199.
References:

[1] M. Elliott, B. Golub and M.O. Jackson, Financial networks and contagion, American Economic Review 104(10), 2014, pp. 3115-3153.

[2] D. Hu, J.L. Zhao, Z. Hua, and M.C.S. Wong, Network-based modeling and analysis of systemic risk in banking systems, MIS $Q$. 36(4), 2012, pp. 1269-1291.

[3] R.N. Mantegna, Hierarchical structure in financial markets, Eur. Phys. J. B 11(1), 1999, pp. 193-197.

[4] J.G. Brida and W.A. Risso, Hierarchical structure of the german stock market, Expert Syst. Appl. 37(5), 2010, pp. 3846-3852.

[5] M. Tumminello, T. Aste, T. Di Matteo and R.N. Mantegna, A tool for filtering information in complex systems, Proceedings of the National Academy of Sciences 102(30), 2005, pp. 1042110426.

[6] J.P. Onnela, A. Chakraborti, K. Kaski, J. Kertész and A. Kanto, Dynamics of market correlations: Taxonomy and portfolio analysis, Phys. Rev. E 68(5), 2003, pp. 056110.

[7] G.-J. Wang, C. Xie and H.E. Stanley, Correlation structure and evolution of world stock markets: Evidence from pearson and partial correlation-based networks, Comput. Econ. 51(3), 2018, pp. 607-635.

[8] D.Y. Kenett, M. Tumminello, A. Madi, G. GurGershgoren, R.N. Mantegna and E. Ben-Jacob, Dominating clasp of the financial sector revealed by partial correlation analysis of the stock market, PLOS ONE 5(12), 2010, pp. 1-14.

[9] D.Y. Kenett, X. Huang, I. Vodenska, S. Havlin and H.E. Stanley, Partial correlation analysis: applications for financial markets, Quantitative Finance 15(4), 2015, pp. 569-578.

[10] G. Bonanno, G. Caldarelli, F. Lillo and R.N. Mantegna, Topology of correlation-based minimal spanning trees in real and model markets, Phys. Rev. E 68, 2003, pp. 046130.

[11] V. Boginski, S. Butenko and P.M. Pardalos, Statistical analysis of financial networks, Computational Statistics \& Data Analysis 48(2), 2005, pp. 431-43.

[12] W.-Q. Huang, X.-T. Zhuang and S. Yao, A network analysis of the chinese stock market, Physica A: Statistical Mechanics and its Applications 388(14), 2009, pp. 2956-2964. 
[13] C.K. Tse, J. Liu and F.C.M. Lau, A network perspective of the stock market, Journal of Empirical Finance 17(4), 2010, pp. 659-67.

[14] V. Boginski, S. Butenko and P.M. Pardalos, Network models of massive datasets, Computer Science and Information Systems 1(1), 2004, pp. 75-89.

[15] J.-P. Onnela, K. Kaski and J. Kertész, Clustering and information in correlation based financial networks, The European Physical Journal B 38(2), 2004, pp. 353-362.

[16] V. Boginski, S. Butenko and P.M. Pardalos, Mining market data: A network approach, Computers \& Operations Research 33(11), 2006, pp. 3171-3184.

[17] F. Emmert-Streib and M. Dehmer, Identifying critical financial networks of the djia: Toward a network-based index, Complexity 16(1), 2010, pp. 24-33.

[18] V. Boginsky, S. Butenko and P.M. Pardalos, On structural properties of the market graph, Innovations in Financial and economic networks, Edward Elgar Publishing Inc., 2003, pp. 29-45.

[19] G.A. Bautin, V.A. Kalyagin, A.P. Koldanov, P.A. Koldanov and P.M. Pardalos, Simple measure of similarity for the market graph construction, Computational Management Science 10(2), 2013, pp. 105-124.

[20] A. Garas and P. Argyrakis, Correlation study of the Athens Stock Exchange, Physica A: Statistical Mechanics and its Applications 380(C), 2007, pp. 399-410.

[21] A. Vizgunov, B. Goldengorin, V. Kalyagin, A. Koldanov, P. Koldanov and P.M. Pardalos, Network approach for the russian stock market, Computational Management Science 11(1), 2014, pp. 45-55.

[22] A. Namaki, A.H. Shirazi, R. Raei and G.R. Jafari, Network analysis of a financial market based on genuine correlation and threshold method, Physica A: Statistical Mechanics and its Applications 390(21), 2011, pp. 3835-41.

[23] G.A. Bautin, V.A. Kalyagin and A.P. Koldanov, Comparative analysis of two similarity measures for the market graph construction Models, Algorithms and Technologies for Network Analysis, Springer, New York, 2013, pp. 29-41.

[24] O. Shirokikh, G. Pastukhov, V. Boginski S. Butenko, Computational study of the US stock market evolution: a rank correlationbased network model, Computational Management Science 10(2-3), 2013, pp. 81-103.
[25] G.-J. Wang, C. Xie, F. Han and B. Sun, Similarity measure and topology evolution of foreign exchange markets using dynamic time warping method: Evidence from minimal spanning tree, Physica A: Statistical Mechanics and its Applications 391(16), 2012, pp. 4136-4146.

[26] V.A. Kalyagin, A.P. Koldanov, P.A. Koldanov P.M. Pardalos, Optimal decision for the market graph identification problem in a sign similarity network, Annals of Operations Research 266(12), 2017, pp. 313-327.

[27] F.X. Diebold and K. Yilmaz, On the network topology of variance decompositions: Measuring the connectedness of financial firms, Journal of Econometrics 182(1), 2014, pp. 119-134.

[28] S. Saavedra, L.J. Gilarranz, R.P. Rohr, M. Schnabel, B. Uzzi and J. Bascompte, Stock fluctuations are correlated and amplified across networks of interlocking directorates, EPJ Data Science. 3(1), 2014, pp. 1-11.

[29] Y. Tang, J.J. Xiong, Z.-Y. Jia and Y.-C. Zhang, Complexities in financial network topological dynamics: Modeling of emerging and developed stock markets, Complexity 2018, 2018, pp. 056110.

[30] J. Birch, A.A. Pantelous K. Soramki, Analysis of Correlation Based Networks Representing DAX 30 Stock Price Returns, Computational Economics 47(4), 2016, pp. 501-525.

[31] P. Caraiani, Characterizing emerging European stock markets through complex networks: From local properties to self-similar characteristics, Physica A: Statistical Mechanics and its Applications 391(13), 2012, pp. 3629-3637.

[32] K. Kosmidou, D. Kousenidis, A. Ladas and C. Negkakis, Determinants of risk in the banking sector during the european financial crisis, Journal of Financial Stability 33, 2017, pp. 285-296.

[33] N. Paltalidis, D. Gounopoulos, R. Kizys and Y. Koutelidakis, Transmission channels of systemic risk and contagion in the European financial network, Journal of Banking \& Finance 61(S1), 2015, pp. 36-52.

[34] M. Affinito and A. Franco Pozzolo, The interbank network across the global financial crisis: Evidence from Italy, Journal of Banking \& Finance 80(C), 2017, pp. 90-107.

[35] G. Iori, G.D. Masi, O.V. Precup, G. Gabbi and G. Caldarelli, A network analysis of the italian overnight money market, Journal of Economic Dynamics and Control 32(1), 2008, pp. 259278. 
[36] H. Chen, Y. Mai S.-P. Li, Analysis of network clustering behavior of the chinese stock market, Physica A: Statistical Mechanics and its Applications 414, 2014, pp. 360-367.

[37] F. Ren and W.-X. Zhou, Dynamic evolution of cross-correlations in the chinese stock market, PLOS ONE 9(5), 2014, pp. 1-15.

[38] B.M. Tabak, D.O. Cajueiro T.R. Serra, Topological properties of bank networks: The case of brazil, International Journal of Modern Physics C 20(08), 2009, pp. 1121-1143.

[39] M.F. da Silva, E.J. de A. Leão Pereira, A.M. da Silva Filho, A.P. Nunes de Castro, J.G.V. Miranda and G.F. Zebende, Quantifying cross-correlation between Ibovespa and Brazilian blue-chips: The DCCA approach, Physica A: Statistical Mechanics and its Applications 424(C), 2015, pp. 124-129.

[40] J. Lee, J. Youn and W. Chang, Intraday volatility and network topological properties in the Korean stock market, Physica A: Statistical Mechanics and its Applications 391(4), 2012, pp. 13541360.

[41] S. Martínez-Jaramillo, B. AlexandrovaKabadjova, B. Bravo-Benitez and J.P. Solórzano-Margain, An empirical study of the mexican banking system's network and its implications for systemic risk, Journal of Economic Dynamics and Control 40, 2014, pp. 242-265.

[42] S. Poledna, J.L. Molina-Borboa, S. MartínezJaramillo, M. van der Leij S. Thurner, The multilayer network nature of systemic risk and its implications for the costs of financial crises, Journal of Financial Stability, vol. 20, 2015, pp. 70 81.

[43] R. Kali and J. Reyes, The architecture of globalization: a network approach to international economic integration, Journal of International Business Studies 38(4), 2017, pp. 595-620.

[44] E. Jondeau, E. Jurczenko and M. Rockinger, Moment component analysis: An illustration with international stock markets, Journal of Business \& Economic Statistics 36(4), 2018, pp. 576-598.

[45] N. Hautsch, J. Schaumburg and M. Schienle, Financial Network Systemic Risk Contributions, Review of Finance 19(2), 2014, pp. 685-738. .

[46] S. Battiston, J.D. Farmer, A. Flache, D. Garlaschelli, A.G. Haldane, H. Heesterbeek,
C. Hommes, C. Jaeger, R. May and M. Scheffer, Complexity theory and financial regulation, Science 351(6275), 2016, pp. 818-819.

[47] E. Nier, J. Yang, T. Yorulmazer and A. Alentorn, Network models and financial stability, Journal of Economic Dynamics and Control 31(6), 2007, pp. 2033-2060.

[48] K. Anand, P. Gai, S. Kapadia and S. Brennan M. Willison, A network model of financial system resilience, Journal of Economic Behavior \& Organization 85, 2013, pp. 219-235.

[49] P. Giudici and A. Spelta, Graphical network models for international financial flows, Journal of Business \& Economic Statistics, 34(1), 2016, pp. 128-138.

[50] I. Androsov, A. Faizliev, E. Korotkovskaya, A. Lunkov, S. Mironov, V. Petrov, S. Sidorov and F. Smolov, Shock diffusion analysis for a directed market network constructed with use of the risk measure $\Delta \mathrm{CoVaR}$, Journal of Physics: Conference Series, 2019.

[51] N. Paltalidis, D. Gounopoulos, R. Kizys and Y. Koutelidakis, Transmission channels of systemic risk and contagion in the european financial network, Journal of Banking \& Finance 61, 2015, pp. 36-52. 